\title{
P-9
}

\section{Phytochemicals and Bioactivities of Cinnamomum Mollissimum}

\author{
Fatin Fasihah Masnon and Farediah Ahmad*
}

Faculty of Science, Universiti Teknologi Malaysia81310 Johor Bahru, Malaysia; E-mail: farediah@kimia.fs.utm.my

Chemical investigation of the crude extracts of the barks of Cinnamomum mollissimum resulted in the isolation of five aporphine alkaloids, namely $N$-methyl-1,2,10-trimethoxyaporphine, $N$-methylhernagine, $N$-methylhernovine, hernagine and hernovine, along with methyl eugenol. The structures of the phytochemicals were elucidated by the basis of spectral analysis. Several bioactivities such as xantine oxidase, antibacterial and antioxidant assays were tested on the extracts and phytochemicals. The screening of xanthine oxidase assay showed that the extracts, hernagine and methyl eugenol have potential inhibition activity.

Keywords: Cinnamomum mollissimum, xantine oxidase, antibacterial, antioxidant. 